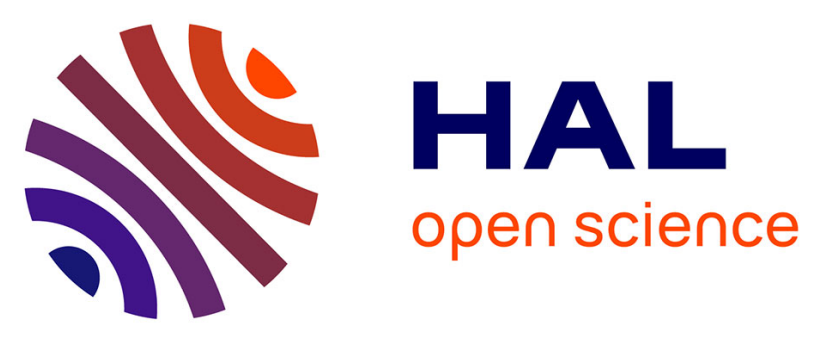

\title{
Tracking and removing modulated sinusoidal components: A solution based on the kurtosis and the Extended Kalman Filter
}

Jean-Luc Dion, Cyrille Stephan, Gaël Chevallier, Hugo Festjens

\section{- To cite this version:}

Jean-Luc Dion, Cyrille Stephan, Gaël Chevallier, Hugo Festjens. Tracking and removing modulated sinusoidal components: A solution based on the kurtosis and the Extended Kalman Filter. Mechanical Systems and Signal Processing, 2013, 38 (2), pp.428-439. 10.1016/j.ymssp.2013.04.001. hal-01911012

\author{
HAL Id: hal-01911012 \\ https://hal.science/hal-01911012
}

Submitted on 24 Nov 2021

HAL is a multi-disciplinary open access archive for the deposit and dissemination of scientific research documents, whether they are published or not. The documents may come from teaching and research institutions in France or abroad, or from public or private research centers.
L'archive ouverte pluridisciplinaire HAL, est destinée au dépôt et à la diffusion de documents scientifiques de niveau recherche, publiés ou non, émanant des établissements d'enseignement et de recherche français ou étrangers, des laboratoires publics ou privés.

\section{(ㅇ)(1) $\$$}

Distributed under a Creative Commons Attribution - NonCommerciall 4.0 International 


\title{
Tracking and removing modulated sinusoidal components: A solution based on the kurtosis and the Extended Kalman Filter
}

\author{
Jean-Luc Dion $^{\mathrm{a}, *}$, Cyrille Stephan ${ }^{\mathrm{b}}$, Gaël Chevallier $^{\mathrm{a}}$, Hugo Festjens ${ }^{\mathrm{a}}$ \\ a Laboratoire d'Ingénierie des Systèmes Mécaniques et des Matériaux (LISMMA) - EA 2336 SUPMECA Paris 3, rue Fernand Hainaut, 93407 \\ Saint Ouen, France \\ b ONERA, Châtillon, France
}

This work describes an automatic method for removing modulated sinusoidal components in signals. The method consists in using the Optimized Spectral Kurtosis for initializing Series of Extended Kalman Filters.

The first section is an introduction to vibration applications with Kalman Filters and modulated sinusoids. The detection process with OSK is described in the second section. The third section concerns the tracking algorithm with SEKF for amplitude and frequency modulated sinusoidal components. The last section deals with the complete process illustrated with an experimental application on a rotating machine.

\section{Introduction}

In the field of Operating Modal Analysis (OMA) applied on structures like helicopters or large structures in energy production, vibration signals are composed with random sources and periodic signals due to rotating machines which cannot be shut down. It is a well-known problem that these harmonic components introduce mistakes in experimental modes extraction by OMA algorithms. The present paper intended to solve this problem by tracking and removing modulated sinusoidal signals in noisy records. The previous work has focused on the detection of sinusoidal components [1]. The present work aims to remove these sinusoidal components by using Series of Extended Kalman Filter [2].

In the field of vibrations, several works have already used Kalman filters for identification, detection, health monitoring or tracking order. In identification and detection processes, Kalman Filters are performed for the localization of cracks on rotating machines [3], for stiffness identification [4] or vibration force estimation [5,6]. Some health monitoring methods for structures and rotating machines are based on Kalman Filters with varying Auto Regressive identification parameters [7-11]. Kalman Filters are also used in active vibration control [12-15] with real-time algorithms and non-stationary signals on smart structures. Other works use Kalman filters to improve time-frequency analysis and order tracking techniques [16-20]. Order Tracking techniques for rotating machines are often based on Vold-Kalman Filter [21,22]. Recent techniques aim to

\footnotetext{
* Corresponding author. Tel.: +3314945 29 12; fax: +33149452910.

E-mail address: jean-luc.dion@supmeca.fr (J.-L. Dion).
} 


\section{Acronyms}

ARMA Auto Regressive - Moving Average

DOF Degree Of Freedom

EKF $\quad$ Extended Kalman Filter
SEKF

OMA

OSK

PBF

PSD
Series of Extended Kalman Filters

Operational Modal Analysis

Optimized Spectral Kurtosis

Pass Band Filter

Power Spectral Density

improve the Vold Kalman Filtering for resampling techniques, high frequency resolution in Fourier analysis and crossing orders detection [23-30]. Most of these works are based on shaft speed information, as an instantaneous frequency is often measured by tachometers. These methods are developed in conjunction with specific signal processing method [31,32].

Promising techniques based of Extended Kalman filtering make the tracking of frequency components possible without tachometer. Different versions of state-space model were proposed to improve robustness and efficiency [33-37], but parameterization is still an open subject.

The focus of this study is based on harmonic components which introduce mistakes in stabilization diagrams used in OMA. Previously, Mohanty and Rixen [38] have shown that "modal analysis procedures fail to identify the modal parameters accurately" in the presence of harmonic excitation.

The whole process of measurement from an excitation source to measured signals is pictured in a diagram (see Fig. 1). Thereafter the structure is supposed to have a linear dynamic behavior. Then in a limited bandwidth, the relation between inputs and outputs can be fully captured by its transfer function $H(s)$, defined in the Laplace domain.

This structure is excited by two kinds of input. The first one is created by a random source which delivers a white noise. Thus it is a stochastic input named $E_{s}(s)$. The second one is created by an unbalanced rotating machine. Contrary to the first input, it supplies a signal whose amplitude and frequency are slowly modulated. As the signal slowly changes over time, it has a phase and is deterministic. It is named $E_{d}(s)$, for deterministic input. Both inputs are not measured.

For a linear structure, the response $Y(s)$ can be separated into its stochastic and deterministic parts:

$$
Y(s)=Y_{s}(s)+Y_{d}(s)
$$

where $Y_{s}(s)=H(s) E_{s}(s)$ is the response due to the stochastic input $E_{s}(s)$ and $Y_{d}(s)=H(s) E_{d}(s)$ is the response due to the deterministic input $E_{d}(s)$.

As errors are unavoidable in measurements, they have to be taken into account too. As for inputs, two kinds of observation noise can distort signals. The first one is a random noise $N_{s}(s)$ which comes from electronic flaws. Its probability law is usually supposed to be Gaussian $p(t)=N(0, W)$ and is characterized in the frequency domain by a flat density of spectral amplitudes, i.e. its energy is uniformly distributed among frequencies.

The second possible noise often results of an electrical field which produces a periodic component at a stable frequency, but with modulated amplitude. Unfortunately this electrical field is also caught by sensors. For instance, in Europe spurious harmonic components at $n * 50 \mathrm{~Hz}$ are well known by experimental engineers. This added noise $N_{d}(f)$ is deterministic and is noticeable in signal spectra as narrow components around a fundamental frequency at $50 \mathrm{~Hz}$ and its harmonics. During modal identification, they look like spurious low-damped modes. Unfortunately, this noise cannot be accurately predicted because it highly depends on each experiment and on each sensor technology. This kind of noise is seldom taken into account, although it can seriously alter signals if modal amplitudes are weak compared to it.

In conclusion, observed signals result in the sum of these noises added to structural responses:

$$
Y_{m}(t)=Y(t)+N_{d}(t)+N_{s}(t)
$$

that can be also separated into its stochastic and deterministic parts:

$$
Y_{m}(t)=Y_{s}(t)+N_{s}(t)+Y_{d}(t)+N_{d}(t)
$$

As a rule, OMA are designed for only stochastic input and noise measurement. It is a strong assumption for these algorithms and is seldom respected. As a result, deterministic components in responses can strongly false results given by OMA algorithms.

The purpose of the proposed method is to identify the deterministic part $Y_{d}(t)$ and to substract it from $Y_{m}(t)$. It will give an approximation of the stochastic response which would be obtained if $E_{d}$ and $N_{d}$ were missing. Then this approximation

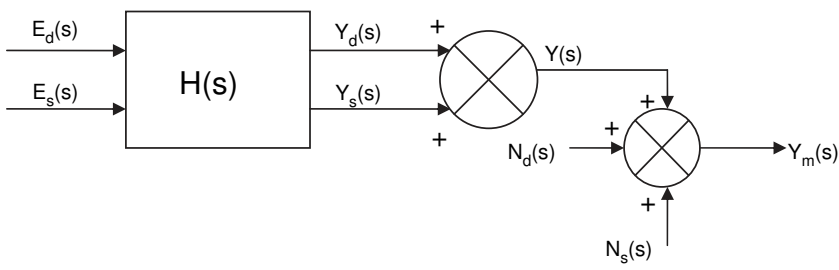

Fig. 1. Block diagram of the studied problem. Measured signals $Y_{m}(\mathrm{~s})$ are composed of stochastic and deterministic components. The aim of the study is to remove the deterministic part in $Y_{m}(\mathrm{~s})$. 
could be possibly used for OMA identification techniques, although here the focus is made on the filtering process. Here the term filtering means that the deterministic component of $Y_{d}(t)$ is going to be filtered from the measured signals $Y_{m}(t)$, without previous knowledge of $Y_{d}(t)$ main frequency.

In the present study, the detection and the tracking of sinusoidal components are performed without the knowledge of frequencies of periodic signal. Studied signals are assumed to be composed of random noise and modulated sinusoidal components. Indeed, measured signals on rotating engines in operational conditions are:

$$
\begin{aligned}
& s(t)=b(t)+\sum_{k} p_{k}(t) \\
& \text { With } b(t)=Y_{s}(t)+N_{s}(t) \text { and } \sum_{k} p_{k}(t)=Y_{d}(t)+N_{d}(t)
\end{aligned}
$$

The first term $b(t)$ is assumed to be mainly composed of modal responses under random excitation. The second terms $p_{k}(t)$ come from engines in operation and from spurious harmonic components of the electric power supply. Signals $p_{k}(t)$ are deterministic and assumed to be both amplitude and frequency modulated. The $k$ th pseudo periodic source is assumed to be composed of $N$ modulated sines:

$$
p_{k}(t)=\sum_{i=1}^{N} a_{i, k}(t) \cos \left(2 \pi i\left(f_{0, k} t+\Delta f_{k} \int_{0}^{t} m_{k}(\theta) d \theta\right)+\varphi_{i, k}\right)
$$

where $a_{i, k}(t)$ is the amplitude for the $k$ th source and the $i$ th sinusoid, $f_{0, k}$ is the central fundamental frequency of the $k$ th source, $\Delta f_{k}$ is the frequency deviation of the $k$ th source, $m_{k}$ is the reduced frequency modulation of the $k$ th source with $\operatorname{MAX}\left\{\left|m_{k}\right|\right\} \leq 1, \varphi_{i, k}$ is the phase of the $k$ th source and the $i$ th sinusoid. In the proposed technique, the frequency modulation is assumed to occur slowly in a short range of variation.

$$
\frac{d}{d t}\left(\Delta f_{k} \int_{0}^{t} m_{k}(\theta) d \theta\right) \ll f_{0, k} \text { with }\left|m_{k}(t)\right|<1 \text { and } \Delta f_{k} \ll f_{0, k}
$$

Two signals are sketched in time domain in Fig. 2: signal A is a frequency and amplitude modulated sinusoid (Fig. 2 upper left) and signal B is a narrow bandwidth random noise (Fig. 2 lower left). Both signals share the same spectrum magnitude (Fig. 2 right), although they have different phase spectras. Thus, for a given PSD or spectrum magnitude where phase information is lost, the original temporal signal cannot be identified: it could be either a modulated sinusoid (deterministic) or a random noise (stochastic).

As a result, in the case of structural behavior identification, dynamic responses based on "output only" techniques have to be free of such modulated sinusoidal signals. Thus, the purpose is to remove sinusoidal components, such as unbalanced rotating machines speed or electrical current components, from the original signal. The method is built upon three main steps.

First, all sinusoidal components are detected with an Optimized Spectral Kurtosis (OSK) [1]. Thus, the number of sinusoidal components and their central frequencies are identified by the OSK. This process is described in Section 2.

Secondly, an Extended Kalman Filter (EKF) is built per detected central frequency. Then a set of EKFs is obtained and called a Series of Extended Kalman Filters (SEKF). Its size is defined by the number of identified frequencies. State variables and variances in the EKF are firstly identified with OSK data in order to initialize the SEKF process. Kalman filtering is a very
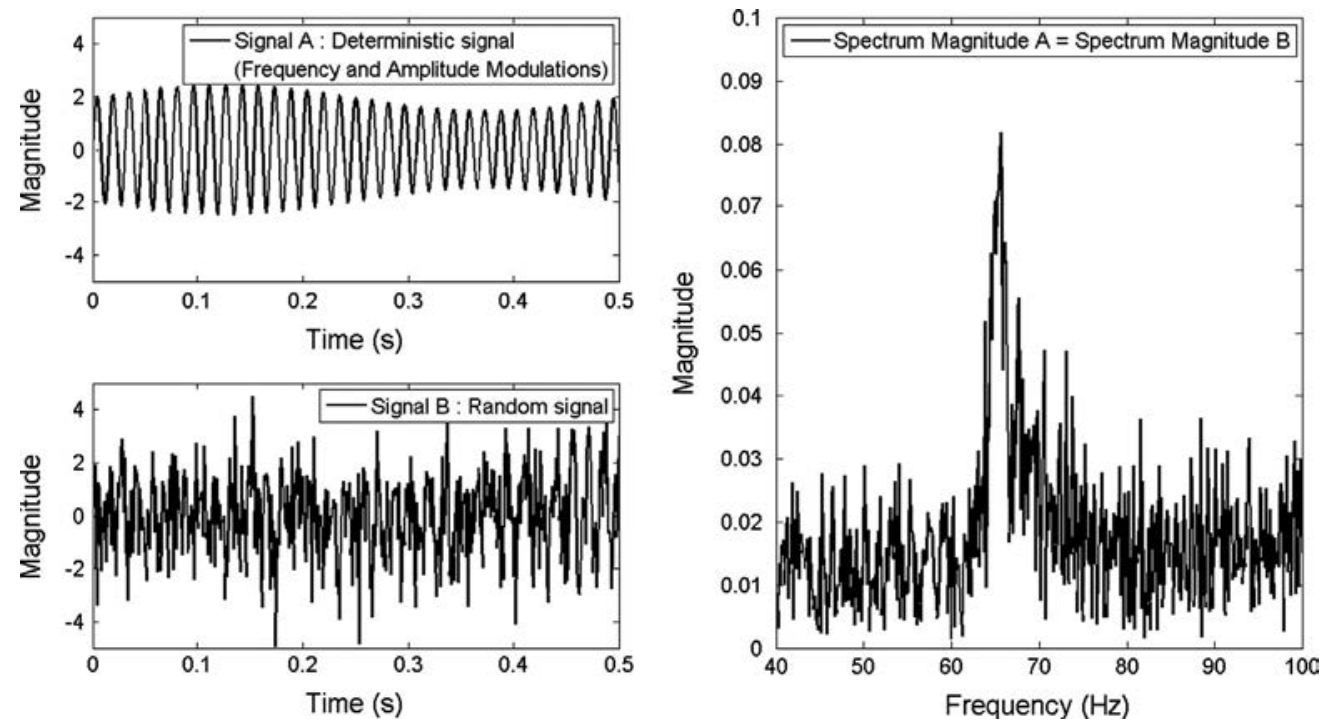

Fig. 2. Example of frequency and amplitude modulations of a sinusoid (Signal A: upper left), random noise in a narrow bandwidth (Signal B: lower left): both signals have the same spectrum magnitude (right) - (signal B is computed with spectrum magnitude of signal A and uniform $0-2 \pi$ random phase). 
powerful tool but it can quickly diverge if state vector, covariance state vector, process noise and observation noise are not properly initialized. In this work, we will focus on the parameterization of Kalman filtering and will demonstrate that appropriate formulas for parameters computation give relevant results. Furthermore, OSK makes possible to perform the initializations of EKF effectively. This step is developed in Section 4.1.

Finally, each detected periodic signal is tracked and removed from the original signal with the help of an EKF. These steps are developed in Section 3 from a theoretical point of view, and numerical applications are detailed in Section 4.

\section{Detection of modulated sinusoidal components}

\subsection{Definition of the optimized spectral kurtosis method}

The detection of sinusoidal components is based on two kinds of information, statistical and spectral, as developed in a previous work [1]. The Optimized Spectral Kurtosis (OSK) is a numerical method applied to signal which provides a spectral description of Kurtosis. It is shortly reminded in this paragraph for the sake of understanding.

Assume that the frequency bandwith of a signal is discretized into $N$ frequency bins. First, the original signal is filtered with a narrow Pass Band Filter (PBF) centered on each frequency bin. As this step is repeated per frequency bin, $N$ filtered signals are obtained.

Secondly, the Kurtosis of each filtered signal is computed and the numerical result defines the assumed nature of the signal (included in the spectral bandwidth). In the $i$ th frequency band, the $i$ th Kurtosis is defined by:

$$
K_{i}=E\left[\left(\frac{X f_{i}-\mu}{\sigma}\right)^{4}\right]
$$

where $X f_{i}$ is the $i$ th filtered signal obtained from the $i$ th PBF, $\mu=E[X], \sigma^{2}=E\left[(X-\mu)^{2}\right]$, and $E(X)$ is the statistical expectation of $X$.

The complete spectral description of Kurtosis is obtained by the $N$ Kurtosis values along the entire frequency bandwidth. In order to obtain an accurate spectral resolution, the used PBF is a sixth order Cauer filter [31] for real-time computation but could also be performed with an ideal PBF for post-processing computation. In the case of a real-time computation, statistical expectations are given by the following linear recursive formula:

$$
E_{k}[Y]=\left(1-\frac{1}{k}\right) E_{k-1}[Y]+\frac{1}{k} y_{k}
$$

where $y_{k}$ is the new data (realization of process $Y$ ), $E_{k-1}[Y]$ the old expectation of $Y$ and $E_{k}$ the new expectation of $Y$.

\subsection{Testing of OSK on an experimental bench}

In order to test OSK and SEKF on a real experiment, a test bench is set up (Fig. 3). The experiment is composed of two masses links with flexible blades. The mechanical system can be described as a two DOF system in the bandwidth $0-100 \mathrm{~Hz}$.

a

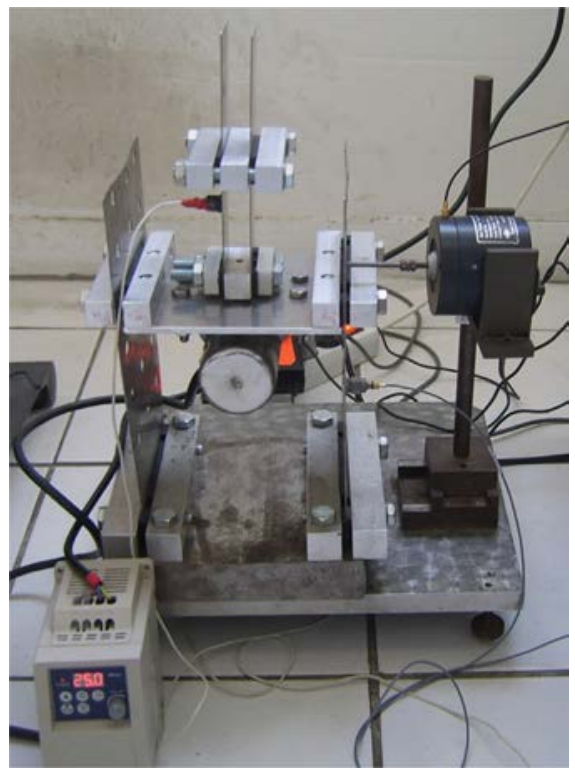

b

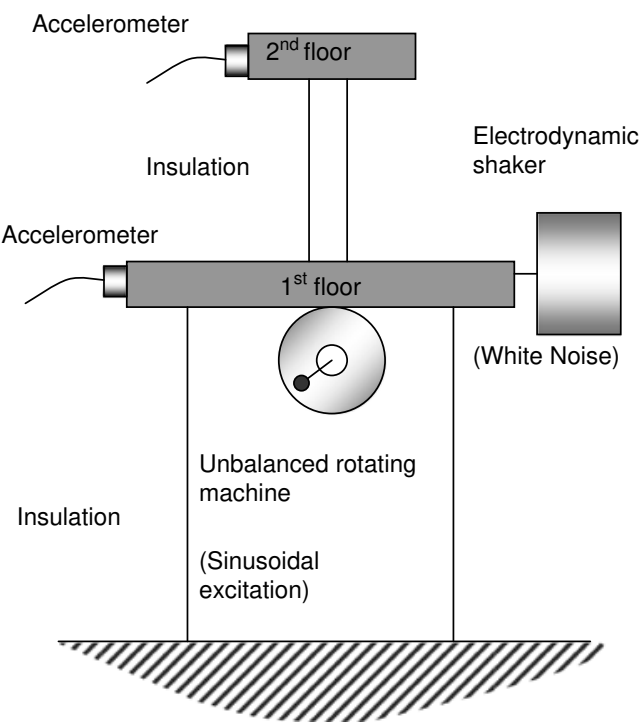

Fig. 3. (a) Picture of the bench test. (b) Diagram of the experiment. 
The system natural frequencies are $9.7 \mathrm{~Hz}$ and $36.7 \mathrm{~Hz}$. The structure is simultaneously excited with a white noise by an electrodynamic shaker and an amplitude and frequency modulated signal thanks to an unbalanced rotating machine closed to $1320 \mathrm{rpm}$ (Fig. 3b). Rotating machine is meaningful of operating system. White noise excitation with Electrodynamic shaker is significant of random excitations such as aerodynamic forces.

Fig. 4 shows PSD and OSK applied on a $131 \mathrm{~s}$ record of the accelerometer of the 1 st floor with $262144\left(2^{18}\right)$ samples. The sampling frequency is $2000 \mathrm{~Hz}$, the filter bandwidth is around $0.15 \mathrm{~Hz}$; detection is defined for a Spectral Kurtosis lower than 2. Sine signal leads to OSK equal to 1.5. Gaussian random signal leads to OSK equal to 3.Detected sinusoidal components are highlighted with "o" markers on both graphs when the OSK is below 2 [1].

The results shown in Fig. 4 highlight expected observations. Frequency $f b=22 \mathrm{~Hz}$ is well detected as a sinusoidal component and not as a structural response. The corresponding OSK at $22 \mathrm{~Hz}$ is lower than 2. Duly, both structural eigenmodes $(9.7 \mathrm{~Hz}$ and $36.7 \mathrm{~Hz}$ ) are not identified as sinusoidal components. Nevertheless, other components, not initially foreseen, are identified as sinusoidal components: the electric network frequency $f r=50 \mathrm{~Hz}$, a low frequency around $5 \mathrm{~Hz}$ and two frequencies: $f r-f b=28 \mathrm{~Hz}$ and $f r+f b=72 \mathrm{~Hz}$.

These components are very small but are parts of the signal. The first one is due to the electric power frequency used for the electric motor. The two following are due to magnitude modulation between the rotation frequency of the engine and its electric power frequency. These components are 100 to 100,000 times smaller than the main excitation at $f b=22 \mathrm{~Hz}$.

Measurements have been made with a piezoelectric accelerometer. Due to this technology, low frequencies are not studied in the present work (even if a $5 \mathrm{~Hz}$ component is detected). The next step consists in removing these components from the original signal.

\section{Tracking of modulated sinusoidal components}

The filtering of modulated sinusoidal components into a composite signal cannot be carried out by classical spectral analysis methods. ARMA filters [31] are not able to separate sinusoidal components and random noise into a same frequency bandwidth. Liftering techniques in cepstral analysis [32] and curve smoothing introduce important distortions in phase spectrum.

As instantaneous phases of modulated signals need to be accurately tracked, here a technique based on Extended Kalman filtering has been selected. Contrary to Vold-Kalman filtering, no shaft speed information is required. Different versions of state-space model have been proposed to model an amplitude and frequency modulated sine for robustness and stability reasons [33-37]. An appropriate state-space model is chosen and detailed in the following paragraph, and formulas for parameterization are proposed.

\subsection{Discrete state space formulation of an amplitude and frequency modulated sinusoid}

The goal of this paragraph is to develop a state space formulation which is relevant on a short time scale. A sinusoid whose amplitude and frequency are modulated over time can be described in the complex domain as an analytic signal:

$$
x(t)=a(t) \exp (j \phi(t))
$$
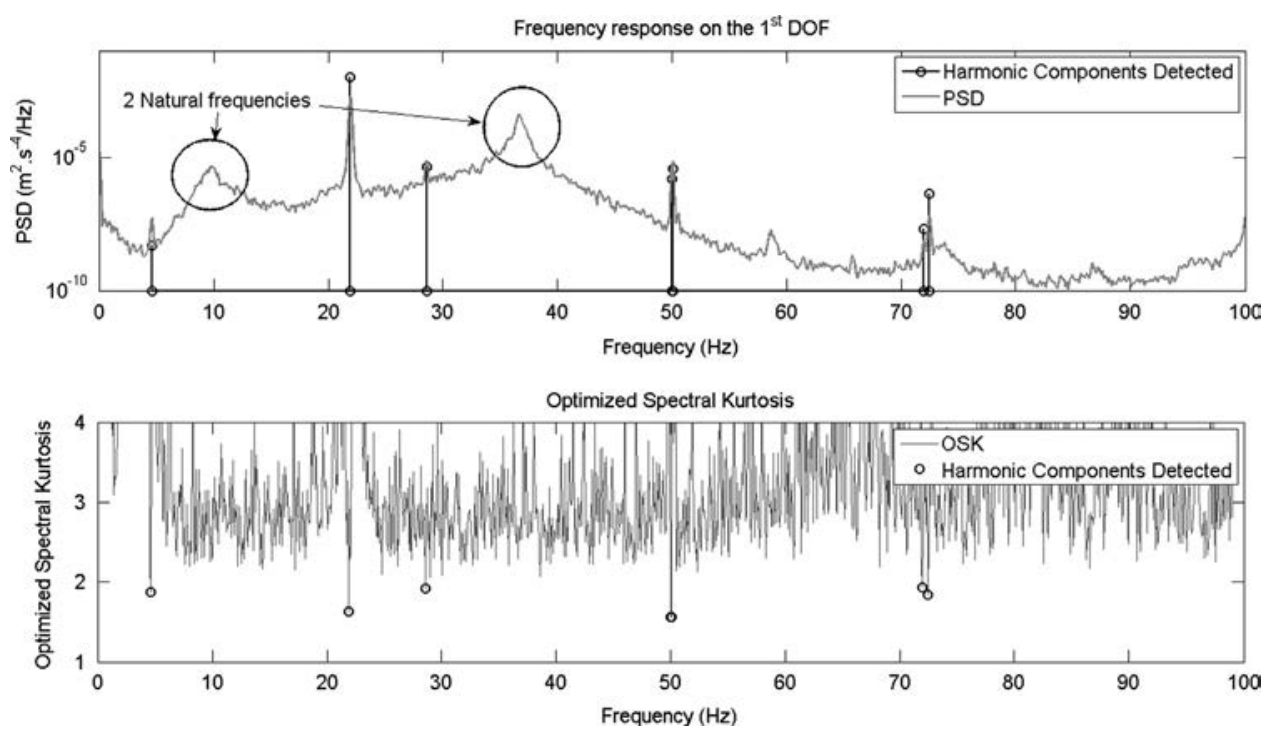

Fig. 4. Detection of sinusoidal components using Spectral Kurtosis. 1 frequency expected but 5 frequencies detected ([1]). 
where $a(t)$ is the instantaneous complex amplitude and $\phi(t)$ is the instantaneous phase. The discrete form of $x(t)$ at time step $t_{n}=\Delta n t$ is $x_{n}=x(\Delta n t)$. The complex variable $x_{n}$ can be divided into its real and imaginary parts $x_{n}=x_{1, n}+j x_{2, n}$. A sinusoid that slightly varies over time can be approximated by:

$$
x_{n}=a_{n} \exp \left(j\left(2 \pi f_{n} n \Delta t\right)\right)
$$

where $f_{n}$ is the instantaneous frequency.

As the parameters $f_{n}$ and $a_{n}$ of the sinusoid slightly vary over time, they are almost equal between two consecutive time steps. Then a transition formulation can be given from $x_{n}$ to $x_{n+1}$ :

$$
x_{n+1} \approx a_{n} \exp \left(j\left(2 \pi f_{n} n \Delta t\right)\right) \times \exp \left(j\left(2 \pi f_{n} \Delta t\right)\right)
$$

This approximation is only true if modulations of $a_{n}$ and $f_{n}$ are slower that the period of the sinusoid. This constraint is assumed to be verified. Then a linear transition is obtained between the imaginary part $x_{2, .}$ and real part $x_{1, .}$ of $x_{n}$ and $x_{n+1}$ :

$$
\begin{aligned}
& x_{1, n+1}=x_{1, n} \cos \left(2 \pi f_{n} \Delta t\right)-x_{2, n} \sin \left(2 \pi f_{n} \Delta t\right) \\
& x_{2, n+1}=x_{1, n} \sin \left(2 \pi f_{n} \Delta t\right)+x_{2, n} \cos \left(2 \pi f_{n} \Delta t\right)
\end{aligned}
$$

or written in a matrix form

$$
\left(\begin{array}{c}
x_{1, n+1} \\
x_{2, n+1}
\end{array}\right)=\left[\begin{array}{cc}
\cos \left(2 \pi f_{n} \Delta t\right) & -\sin \left(2 \pi f_{n} \Delta t\right) \\
\sin \left(2 \pi f_{n} \Delta t\right) & \cos \left(2 \pi f_{n} \Delta t\right)
\end{array}\right]\left(\begin{array}{c}
x_{1, n} \\
x_{2, n}
\end{array}\right)
$$

The instantaneous amplitude $a_{n}$ is given by:

$$
a_{n}=\sqrt{x_{1, n}^{2}+x_{2, n}^{2}}
$$

As $a_{n}$ and $f_{n}$ should be allowed to vary over time, it is proposed here to use the following non-linear state space formulation:

$$
\mathrm{X}_{n+1}=\phi\left(\mathrm{X}_{n}\right)+\mathrm{W}_{n}
$$

where $X_{n}=\left(x_{1, n} x_{2, n} x_{3, n}\right)^{T}$ and $W_{n}$ the process noise. A state variable $x_{3, n}=2 \pi f_{n} \Delta t$ was added to track the evolution of the instantaneous frequency $f_{n}$. The transition between two time steps is composed by a sum of two parts: the stationary part and the evolutionary part.

The stationary part links two successive points of a stationary sinusoid by $\phi($.$) . Then \phi($.$) is assumed as the non-linear$ transition function and is given by $\phi\left(X_{n}\right)=F_{n} X_{n}$ : where

$$
F_{n}=\left(\begin{array}{ccc}
\cos \left(x_{3, n}\right) & -\sin \left(x_{3, n}\right) & 0 \\
\sin \left(x_{3, n}\right) & \cos \left(x_{3, n}\right) & 0 \\
0 & 0 & 1
\end{array}\right)
$$

The two first components are related to the complex amplitude and are obtained by the previous linear relation. The third component $x_{3, n+1}=x_{3, n}$ constrains the frequency not to change strongly between two time steps.

Up to now, the non-stationary behavior of the sinusoid was not modeled because it is not possible to formulate an exact equation for its evolution. We suppose that $W_{n}$ is a random variable whose probability law is Gaussian: $W_{n}=N(0, Q)$, where $Q$ is its variance matrix. Then variations of amplitude and frequency are allowed by random values of $W_{n}$.

In a first glance, it could seem strange to choose a random variable for an effect which is generally deterministic. For instance, the variation of frequency excitation due to an engine is mainly deterministic. Anyway, this state space does not need to represent accurately the evolution of a sinusoid on a long period, but only step by step. Then on a short time scale, a random evolution of $a_{n}$ and $f_{n}$ is enough to model a non-stationary sinusoid.

For a signal composed of $M$ modulated sinusoidal components, the size of the state function is $3 \mathrm{M}$.

$$
F_{n}\left(X_{n}\right)=\left[\begin{array}{ccccccc}
\cos \left(x_{3, n}\right) & -\sin \left(x_{3, n}\right) & 0 & \ldots & 0 & 0 & 0 \\
\sin \left(x_{3, n}\right) & \cos \left(x_{3, n}\right) & 0 & \ldots & 0 & 0 & 0 \\
0 & 0 & 1 & & 0 & 0 & 0 \\
\vdots & \vdots & & \ddots & 0 & 0 & 0 \\
0 & 0 & 0 & \ldots & \cos \left(x_{3+3(M-1), n}\right) & -\sin \left(x_{3+3(M-1), n}\right) & 0 \\
0 & 0 & 0 & \ldots & \sin \left(x_{3+3(M-1), n}\right) & \cos \left(x_{3+3(M-1), n}\right) & 0 \\
0 & 0 & 0 & \ldots & 0 & 0 & 1
\end{array}\right] \text { and } X_{n}=\left(\begin{array}{c}
x_{1, n} \\
x_{2, n} \\
x_{3, n} \\
\vdots \\
x_{1+3(M-1), n} \\
x_{2+3(M-1), n} \\
x_{3+3(M-1), n}
\end{array}\right)
$$

This state space formulation is nonlinear: the transition function $\phi\left(X_{n}\right)$ is varying over time and depends on the frequency modulation.

In reality, only the real part $x_{1, n}$ of the analytic signal $x_{n}$ can be observed. Unlike the transition phase, the observation phase is completely linear

$$
\begin{aligned}
Z_{n} & =\left[\begin{array}{lll}
1 & 0 & 0
\end{array}\right] X_{n}+V_{n} \\
Z_{n} & =x_{1, n}+V_{n}
\end{aligned}
$$


for one sinusoid and slightly more complex for $M$ sinusoids

$$
\begin{aligned}
Z_{n} & =\left[\begin{array}{lllllll}
1 & 0 & 0 & \ldots & 1 & 0 & 0
\end{array}\right] X_{n}+V_{n} \\
Z_{n} & =x_{1, n}+\ldots+x_{1+3(M-1) n}+V_{n}
\end{aligned}
$$

where $V_{n}$ is a noise observation random process.

Finally, a nonlinear discrete state space model has been formulated to model the transition and observation of sinusoid components mixed with random processes

$$
\left\{\begin{array}{l}
X_{n+1}=\phi\left(X_{n}\right)+W_{n} \\
Z_{n+1}=H X_{n+1}+V_{n+1}
\end{array}\right.
$$

where $\phi($.$) is the nonlinear transition function given by \phi\left(X_{n}\right)=F\left(X_{n}\right) X_{n}$ and $H($.$) is the observation matrix given by:$

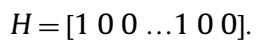

\subsection{Application to the Extended Kalman Filter}

Kalman filtering refers to a family of algorithms that track the temporal evolution of a dynamic model based on noised measurements:

$$
\left\{\begin{array}{l}
X_{n+1}=f\left(X_{n}, W_{n}\right) \\
Z_{n+1}=h\left(X_{n+1}, V_{n+1}\right)
\end{array}\right.
$$

described here in discrete time domain. An efficient algorithm in terms of means and covariances can be derived when $f($. and $h($.) are linear. Indeed it estimates the state probability distribution by its two first moments. Unfortunately, they are no longer sufficient to characterize the distribution in the nonlinear case. Then some approximations have to be done in order to find a practical algorithm.

The extended Kalman filtering is an adaptation of the classical Kalman filtering to problems with state dynamics governed by nonlinear transformations. Although it is not required here, it should be noticed that it can also handle a nonlinear transformation from state variables to measurement variables. It generally exhibits a good robustness because it uses linear approximation over small ranges of state space. Without any input control, the state model is given by the first equation in system (22) where $W_{n}$ is the process noise assumed to be Gaussian $N(0, Q)$. The observation model is described by the second equation in system (22) where $H X_{n+1}$ is the observation function and $V_{n+1}$ the observation noise assumed to be Gaussian $N(0, R)$. State and observation noises are assumed to be uncorrelated. The Extended Kalman Filter [2] is defined using predict and update phases. The predict phase gives an a priori estimate of the state and covariance based on previous time step $t_{n}$ :

$$
\text { Predicted state } \hat{X}_{n+1 \mid n}=\hat{F}_{n \mid n} \hat{X}_{n \mid n}
$$

Predicted estimated covariance $\hat{P}_{n+1 \mid n}=\tilde{F}_{n \mid n} \hat{P}_{n \mid n} \tilde{F}_{n \mid n}^{T}+Q$

And the update phase corrects the deviation of these estimations based on new observation at time step $t_{n+1}$ :

Innovation $\quad \tilde{Y}_{n+1 \mid n}=Z_{n+1}-H \hat{X}_{n+1 \mid n}$

Innovation covariance $\quad S_{n+1}=H \hat{P}_{n+1 \mid n} H^{T}+R$

Kalmangain

$$
K_{n+1}=\hat{P}_{n+1 \mid n} H^{T}\left(S_{n+1}\right)^{-1}
$$

Updated state estimate $\quad \hat{X}_{n+1 \mid n+1}=\hat{X}_{n+1 \mid n}+K_{n+1} \tilde{Y}_{n+1 \mid n}$

Updated estimate covariance $\quad \hat{P}_{n+1 \mid n+1}=\left(I-K_{n+1} H\right) \hat{P}_{n+1 \mid n}$

As the transition function $\phi$ is non-linear but differentiable, it is well locally approximated thanks to its Jacobian:

$$
\tilde{F}_{n \mid n}=\left.\left(\nabla_{X}(F(X) X)^{T}\right)^{T}\right|_{X=\hat{X}_{n \mid n}}
$$

In the case of $M$ modulated components, the first order derivative is required:

$$
\nabla X_{n}=\left(\frac{\partial}{\partial X_{1, n}} \frac{\partial}{\partial X_{2, n}} \cdots \frac{\partial}{\partial X_{3 M,-1 n}} \frac{\partial}{\partial X_{3 M, n}}\right)^{T}
$$


and the approximation becomes a banded block matrix:

$$
\tilde{F}_{n}=\left[\begin{array}{cccc}
\tilde{F}_{n, 1} & 0 & \ldots & 0 \\
0 & \tilde{F}_{n, 2} & \ddots & \vdots \\
\vdots & \ddots & \ddots & 0 \\
0 & \ldots & 0 & \tilde{F}_{n, M}
\end{array}\right]
$$

where each elementary block is given by:

$$
\tilde{F}_{n, i}=\left[\begin{array}{ccc}
\cos \left(\hat{x}_{3 i, n}\right) & -\sin \left(\hat{x}_{3 i, n}\right) & -\hat{x}_{1+3(i-1), n} \sin \left(\hat{x}_{3 i, n}\right)-\hat{x}_{2+3(i-1), n} \cos \left(\hat{x}_{3 i, n}\right) \\
\sin \left(\hat{x}_{3 i, n}\right) & \cos \left(\hat{x}_{3 i, n}\right) & \hat{x}_{1+3(i-1), n} \cos \left(\hat{x}_{3 i, n}\right)-\hat{x}_{2+3(i-1), n} \sin \left(\hat{x}_{3 i, n}\right) \\
0 & 0 & 1
\end{array}\right]
$$

This state matrix is composed of $3 \mathrm{M}$ equations which could be described as $M$ independent systems of 3 equations. The complete EKF can be expanded in a series of $M$ elementary EKFs which could be computed in a same step or in $M$ independent steps. This point of view allows parallel computation of all EKF cells. The complete process is described in the block diagram in next section, Fig. 5.

\section{OSK and SEKF in operation}

In this section, the aim is to propose a method for the filtering process of experimental signals as described in the block diagram (Fig. 5).

The filtering process consists in removing detected and tracked sinusoidal components. The efficiency of the method is due to the fact that OSK allows an accurate initialization of SEKF. The combination of OSK and SEKF techniques could be

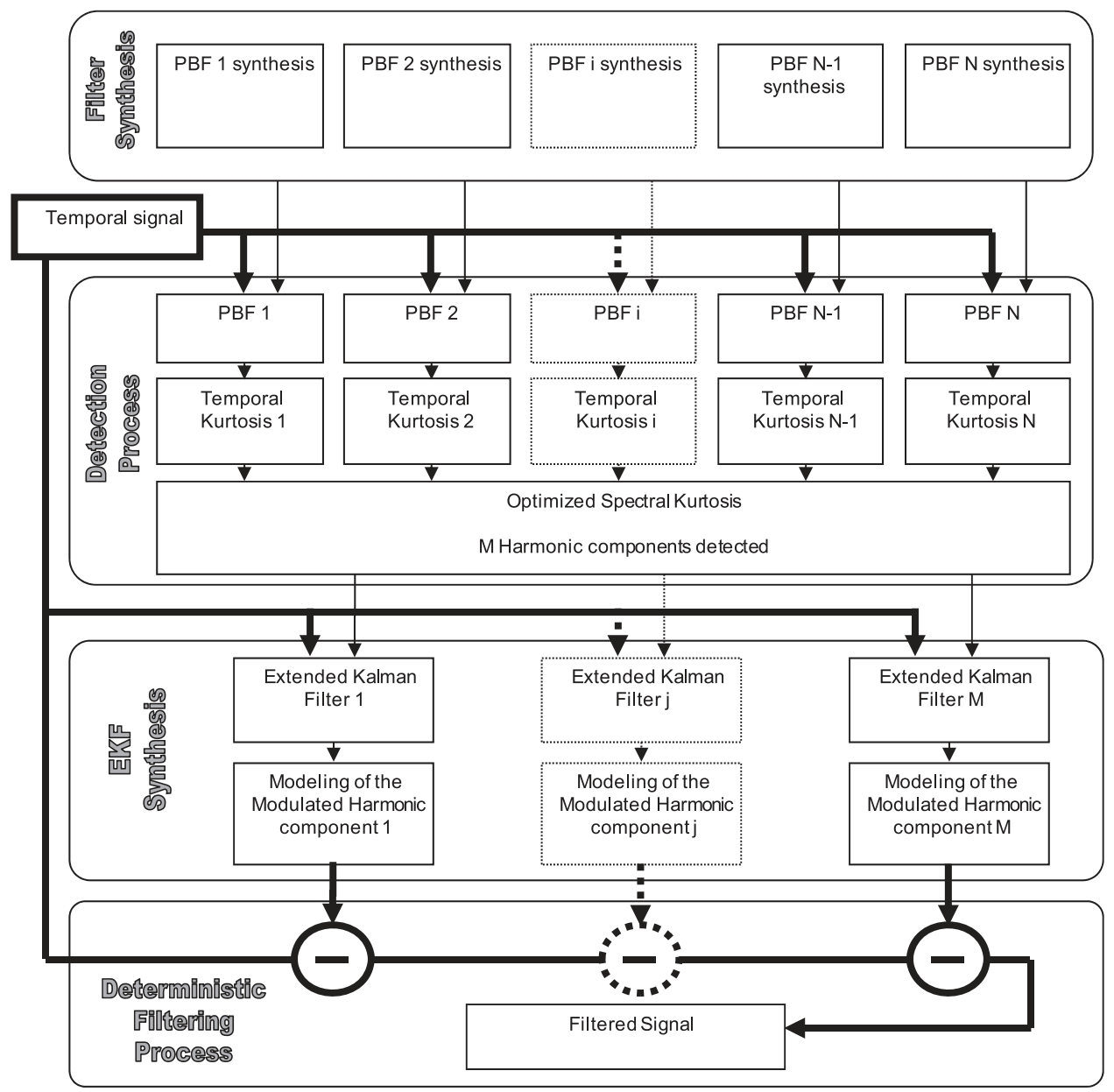

Fig. 5. Block diagram of the method. Four main steps in filtering process:. filter synthesis ( $\mathrm{N}$ filters computed off line), sinusoidal components detection with OSK (real time or post-process of M components), Extended Kalman filtering (real time or post-process of M Kalman Filters), filtering process (real time or post-process of M modulated sinusoids). 
performed into two ways, depending whether real time or post-processing computation is required. These two ways are illustrated in Fig. 6. The real time computation is performed for ARMA filtering with finite difference equations:

$$
y_{n}=\sum_{i=0}^{N} b_{i} x_{n-i}+\sum_{j=0}^{M} a_{j} x_{n-j}
$$

with output vector $\mathrm{y}$, input vector $\mathrm{x}$, and ARMA filter coefficients vectors a and $\mathrm{b}$.

Real time OSK computation is performed with means (expectations) computed in a recursive sense for all statistical momentum (see Eq. (8)). At last, Kalman filters are in nature designed for real time computation.

In order to test the method, the previous Series of EKF have been applied on signals obtained with the test bench described in Sections 2.1 and 2.2. Previous results obtained in Section 2.2 have highlighted 4 modulated components. The tracking of two of them is presented in this section. The first frequency $(22 \mathrm{~Hz})$ corresponds to the engine rotation and is amplitude and frequency modulated. The second frequency corresponds to the electric power frequency ( $50 \mathrm{~Hz}$ ) and is only amplitude modulated.

\subsection{Initializing the series of Extended Kalman Filters}

An important step for EKF is the estimation of initial parameters conditions. The first unknown parameter is the suitable size of the SEKF. In a second step, for each EKF, several parameters and their variances have to be estimated. The efficiency of the filtering process strongly depends on the quality of estimated initial parameters.

For each EKF, initial values of nine parameters have to be set:

- three parameters used for sinusoidal component modeling,

- three parameters variances (one per parameter),

- three process noise variances (one per parameter).

The observation noise variance is defined for the complete SEKF.

For the proposed filtering method based on a SEKF composed of $M$ EKF, $9 \mathrm{M}+2$ initial conditions have to be estimated. The very first parameter is the number $M$ of EKF. This parameter is defined by OSK and equal to the number of narrow bandwidths in which detection occurs. The second one is the observation noise variance. The three parameters used to define each sinusoidal component depend on the amplitude, the frequency and the phase of this component.

The estimated amplitude $\tilde{A}_{j}$ of the $j$ th detected component is determined from the DSP $\gamma_{k}$ or the FFT $s_{k}$ results:

$$
\tilde{A}_{j} \cong 2 s_{k \mid f_{j}=f_{k}} \cong \sqrt{\Delta f \gamma_{k \mid f_{j}=f_{k}}} .
$$

These two estimators overestimate the actual value of $A$. The higher the frequency is modulated, the larger the overestimation.

The estimated frequency $\tilde{f}_{j}$ is determined from the OSK and equal to the center frequency $f_{k}$ of the narrow bandwidth $\Delta f_{j}$ in which the detection occurs.

The phase is not estimated and always set in the present method to 0 . This lack of estimation is quickly vanished by the specific efficiency of the Kalman method for this kind of parameter [17].

The $j$ th sinusoidal component modeling is also estimated:

$$
\left\{\begin{array}{l}
x_{1+3(j-1), 0}=\tilde{A}_{j} \\
x_{2+3(j-1), 0}=0 \\
x_{3+3(j-1), 0}=\Delta t 2 \pi f_{j}
\end{array}\right.
$$

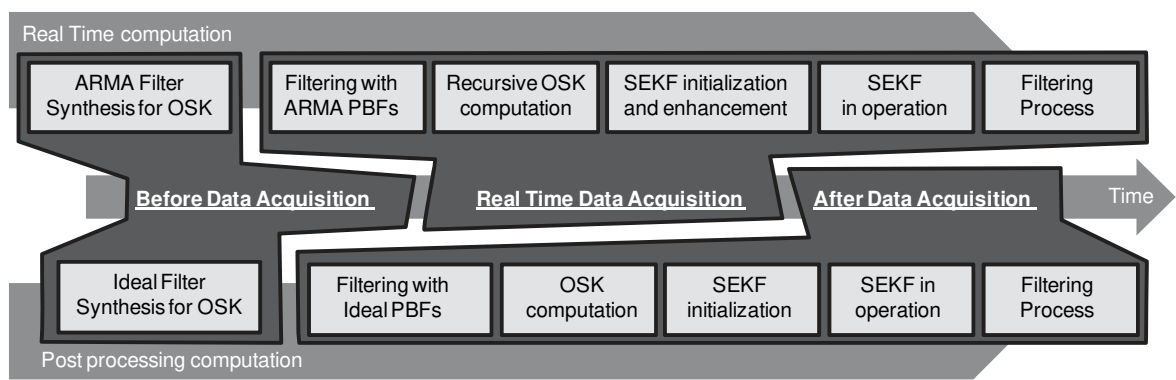

Fig. 6. Chronogram of two different ways for combining and computing OSK and SEKF: real-time and post-processing computation. 
Due to the statistical properties of sinus functions, the estimated variances $\tilde{\sigma}_{1+3(j-1)}^{2}, \tilde{\sigma}_{2+3(j-1)}^{2}$ of the first two parameters are:

$$
\left\{\begin{array}{l}
P_{1+3(j-1), 0}=\sigma_{1+3(j-1), 0}^{2}=\frac{1}{2} \tilde{A}_{j}^{2} \\
P_{2+3(j-1), 0}=\sigma_{2+3(j-1), 0}^{2}=\frac{1}{2} \tilde{A}_{j}^{2}
\end{array}\right.
$$

These estimators underestimate actual variances. The larger the amplitude modulation, the bigger the variance underestimation. For robustness reasons in numerical applications, these parameters are chosen four times bigger.

The estimation of the third variance $\tilde{\sigma}_{1+3(j-1)}^{2}$ is based on the bandwidth $\Delta f_{j}$ in which the detection $j$ occurs:

$$
P_{3+3(j-1), 0}=\sigma_{3+3(j-1), 0}^{2}=\left(2 \pi \Delta f_{j} \Delta t\right)^{2}
$$

The two first process noise variances $Q_{1+3(j-1)}$ and $Q_{2+3(j-1)}$ tend to zero since the modeling of amplitude modulated component is linear. The third process noise variance deals with the frequency modulation and depends on the "velocity" of the frequency modulation. The chosen estimation is:

$$
\left.Q_{3+3(j-1)}=\frac{2 \pi \Delta f_{j} \Delta t}{f_{j} T}\right)^{2}
$$

This approximation can be seen as a linear frequency evolution along $\Delta \mathrm{f}_{\mathrm{j}}$ during the observation time $T$.

The observation noise variance $R$ is simply chosen equal to the observed signal variance. This approximation is relevant as long as the studied signal is mainly stochastic, i.e. the energy of sinusoid components is weak compared to the total signal energy

\subsection{Numerical results and filtering efficiency}

The SEKF was performed on the experiment with two tracked sinusoids previously detected by the OSK. The first component is around $22 \mathrm{~Hz}$ and the second around $50 \mathrm{~Hz}$. For each component, the amplitude modulation is determined with the instantaneous amplitude:

$$
a_{n}=\sqrt{x_{1, n}^{2}+x_{2, n}^{2}}
$$

This amplitude could also be obtained by the complex analytic signal composed of the observed signal (real part) and its Hilbert transform (imaginary part).

The frequency modulation is computed by the instantaneous frequency:

$$
f_{n}=\frac{x_{3, n}}{2 \pi \Delta t}
$$

The SEKF provides an analytic signal $\left\{x_{1, n}, x_{2, n}\right\}$ per sinusoid from the original signal. Thus one tracked signal is obtained per sinusoid. Spectral amplitudes of these tracked signals show the main components at 22 and $50 \mathrm{~Hz}$ and lateral modulated bands (Fig. 7).

The first tracked signal concerns the effect of engine rotation. It is characterized by its frequency and amplitude modulations. As it can be observed on the Fig. 8, its instantaneous frequency fluctuates between 21.8 and $22 \mathrm{~Hz}$.

The second tracked signal is due to the electrical network and is only amplitude-modulated. This property could have been foreseen since the $50 \mathrm{~Hz}$ component due to network is absolutely stable in frequency.

The very last step, the effective filtering process, is simply performed by removing the tracked signals from the original signal in time domain. Fig. 9 shows spectral amplitudes before and after the filtering process for two tracked components $(22 \mathrm{~Hz}$ and $50 \mathrm{~Hz}$ ). Although the filtering process removes the $50 \mathrm{~Hz}$ component well, it does not show the same behavior on the $22 \mathrm{~Hz}$ component. This lower efficiency could be explained by the frequency evolution of $22 \mathrm{~Hz}$ which makes the tracking harder. However, this component is twenty times lower after the filtering process, which is still a significant improvement.

\section{Conclusion}

An important difficulty in the application of Kalman filters is the initialization of state variables and variances. The most important asset of OSK is to initialize the computation of SEKF. The combination of Optimized Spectral Kurtosis and Series of Extended Kalman Filters allows a robust computational technique to track and remove sinusoidal components from signal. The filtering process can be performed during or after real time data acquisition. The complete signal filtering can be performed automatically, even if the size of SEKF is defined by OSK. According to our numerical and experimental tests, the tested method is limited by frequency modulation rate. Those limits are a new challenge for our work in progress, especially for non-smooth nonlinear systems. 


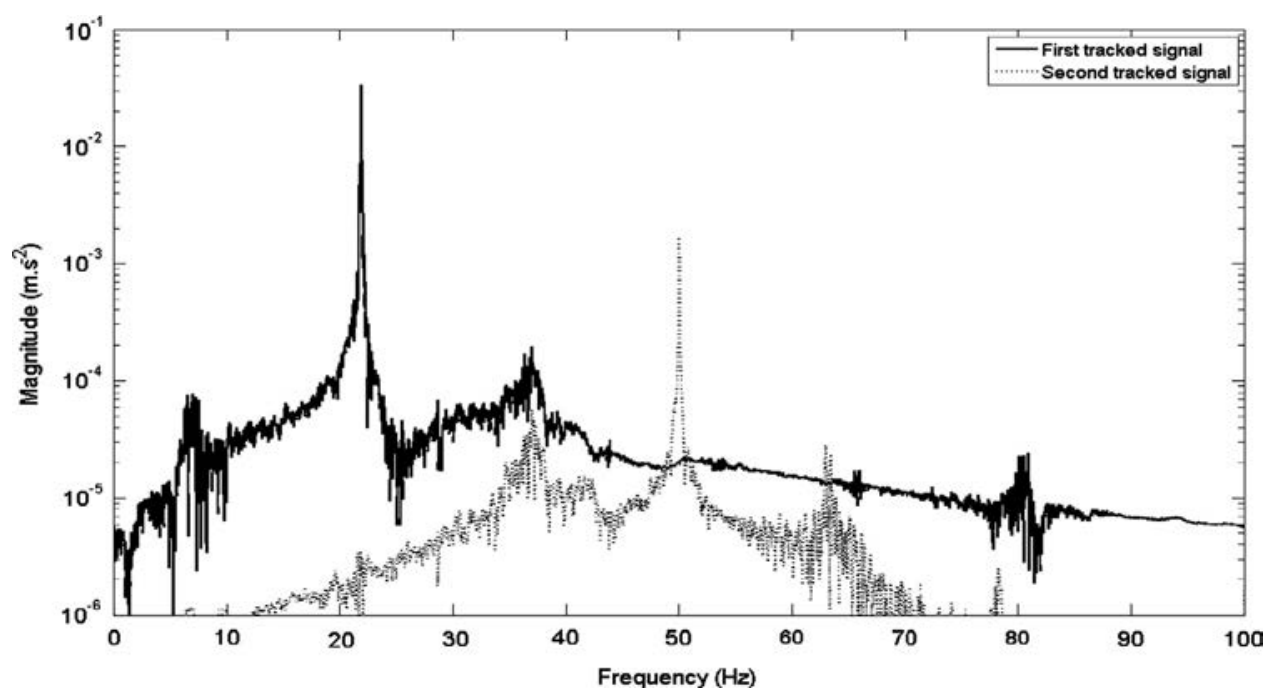

Fig. 7. Spectra of two tracked signals. Symmetric lateral bands are due to modulation effects on spectra.

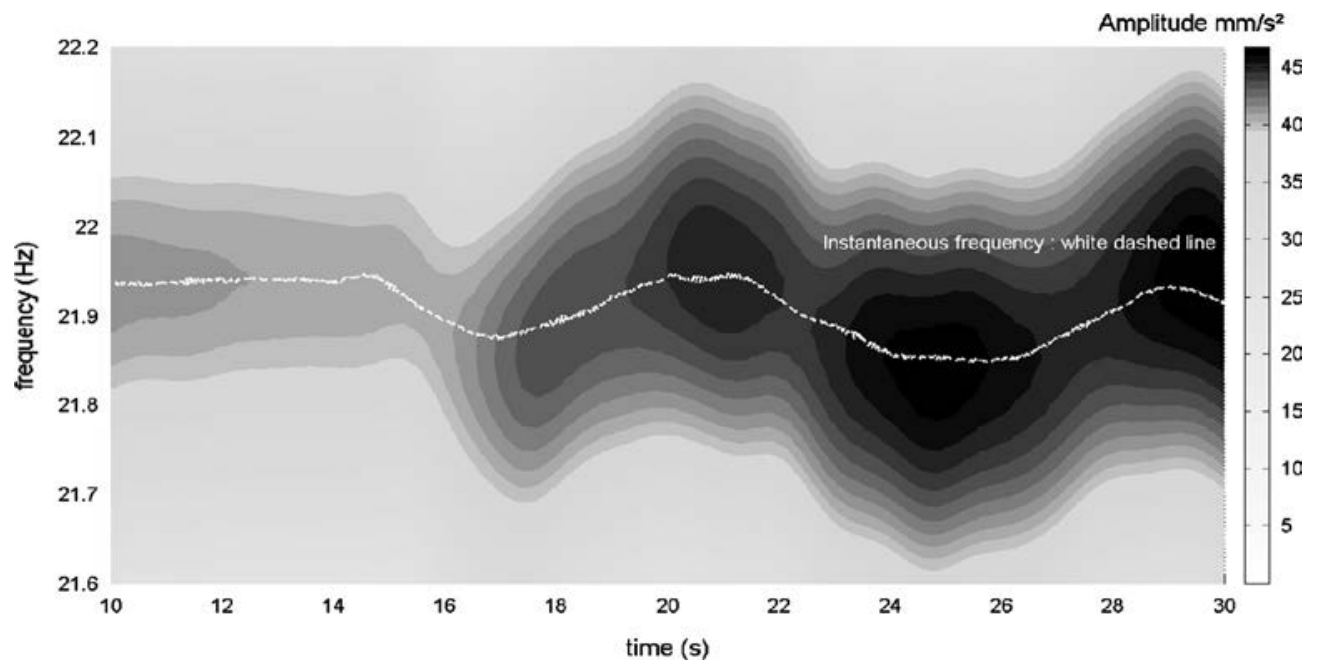

Fig. 8. Time-frequency representation of the first tracked signal. Magnitude and frequency modulations are identified with a good accuracy. (Instantaneous frequency is identified with 4 significant digits).

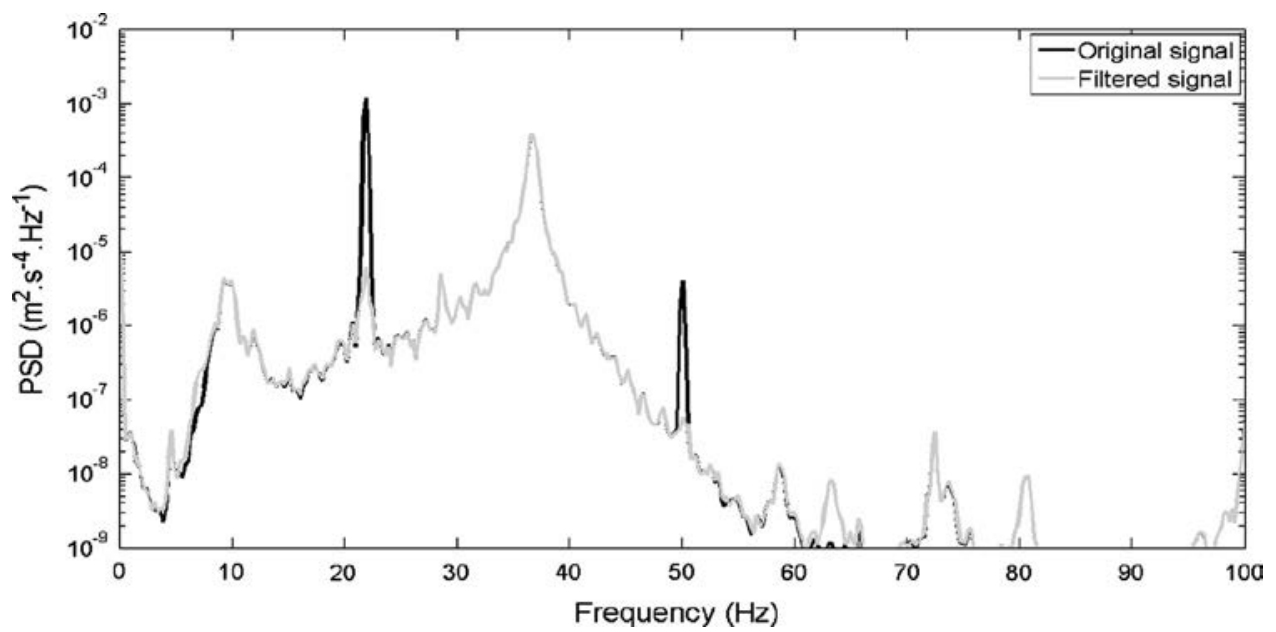

Fig. 9. Spectra before and after the filtering process. The two modulated sinusoidal components are successfully removed. 


\section{References}

[1] J.-L. Dion, I. Tawfiq, G. Chevallier, Sinusoidal component detection: optimized Spectral Kurtosis for operational modal analysis, Mech. Syst. Signal Process. 26 (2012) 24-33.

[2] R.E. Kalman, A new approach to linear filtering and prediction Problems, Trans. Am. Soc. Mech. Eng.-Ser. D J. Basic Eng. 82 (1960) $35-45$.

[3] S. Seibold, K. Weinert, A time domain method for the localization of cracks in rotors, J. Sound Vib. 195 (1996) 57-73.

[4] Z. Wang, W. Yan, Y. Shao, Three-step method for stiffness identification of inter-story shearing structures under ambient excitation, Tsinghua Sci. Technol. 14 (2009) 69-74.

[5] C.-K. Ma, D.-C. Lin, J.-M. Chang, Estimation of forces generated by a machine mounted upon isolators under operating conditions, J. Franklin Inst. 336 (1999) 875-892.

[6] C.-K. Ma, C.-C. Ho, An inverse method for the estimation of input forces acting on non-linear structural systems, J. Sound Vib. 275 (2004) 953-971.

[7] Y.M. Zhan, A.K.S. Jardine, Adaptive autoregressive modeling of non-stationary vibration signals under distinct gear states. Part 1: modeling, J. Sound Vib. 286 (2005) 429-450.

[8] Y. Zhan, C.K. Mechefske, Robust detection of gearbox deterioration using compromised autoregressive modeling and Kolmogorov-Smirnov test statistic-Part I: Compromised autoregressive modeling with the aid of hypothesis tests and simulation analysis, Mech. Syst. Signal Process. 21 (2007) 1953-1982.

[9] Y. Zhan, C.K. Mechefske, Robust detection of gearbox deterioration using compromised autoregressive modeling and Kolmogorov-Smirnov test statistic. Part II: experiment and application, Mech. Syst. Signal Process. 21 (2007) 1983-2011.

[10] Y. Shao, C.K. Mechefske, Gearbox vibration monitoring using extended Kalman filters and hypothesis tests, J. Sound Vib. 325 (2009) $629-648$.

[11] G. Wang, Z. Luo, X. Qin, Y. Leng, T. Wang, Fault identification and classification of rolling element bearing based on time-varying autoregressive spectrum, Mech. Syst. Signal Process. 22 (2008) 934-947.

[12] I.T. Al-Zaharnah, Suppressing vibrations of machining processes in both feed and radial directions using an optimal control strategy: the case of interrupted cutting, J. Mater. Process. Technol. 172 (2006) 305-310.

[13] X.-J. Dong, G. Meng, J.-C. Peng, Vibration control of piezoelectric smart structures based on system identification technique: Numerical simulation and experimental study, J. Sound Vib. 297 (2006) 680-693.

[14] J. Marzbanrad, G. Ahmadi, H. Zohoor, Y. Hojjat, Stochastic optimal preview control of a vehicle suspension, J. Sound Vib. 275 (2004) $973-990$.

[15] T. Yoshimura, K. Edokoro, N. Ananthanarayana, An Active suspension model for rail/vehicle systems with preview and stochastic optimal control, J. Sound Vib. 166 (1993) 507-519.

[16] M. Bai, J. Huang, M. Hong, F. Su, Fault diagnosis of rotating machinery using an intelligent order tracking system, J. Sound Vib. 280 (2005) 699-718.

[17] J.R. Blough, Development and analysis of time variant discrete Fourier transform order tracking, Mech. Syst. Signal Process. 17 (2003) 1185-1199.

[18] Y. Guo, K.K. Tan, Order-crossing removal in Gabor order tracking by independent component analysis, J. Sound Vib. 325 (2009) $471-488$.

[19] M.-C. Pan, C.-C. Chiu, Investigation on improved Gabor order tracking technique and its applications, J. Sound Vib. 295 (2006) $810-826$.

[20] M.-C. Pan, S.-W. Liao, C.-C. Chiu, Improvement on Gabor order tracking and objective comparison with Vold-Kalman filtering order tracking, Mech. Syst. Signal Process. 21 (2007) 653-667.

[21] H. Vold, J. Leuridan, High Resolution Order Tracking at Extreme Slew Rates, Using Kalman Tracking Filters. SAE Paper No. $931288,1993$.

[22] H. Vold, H. Herlufsen, Multi axle order tracking with the Vold-Kalman tracking filter, Sound Vib. 31 (5) (1997) 30-34.

[23] Y. Guo, K.K. Tan, High efficient crossing-order decoupling in Vold-Kalman filtering order tracking based on independent component analysis, Mech. Syst. Signal Process. 24 (2010) 1756-1766.

[24] M.-C. Pan, Y.-F. Lin, Further exploration of Vold-Kalman-filtering order tracking with shaft-speed information-I: Theoretical part, numerical implementation and parameter investigations, Mech. Syst. Signal Process. 20 (2006) 1134-1154.

[25] M.-C. Pan, Y.-F. Lin, Further exploration of Vold-Kalman-filtering order tracking with shaft-speed information-II: Engineering applications, Mech. Syst. Signal Process. 20 (2006) 1410-1428.

[26] M.-C. Pan, C.-X. Wu, Adaptive Vold-Kalman filtering order tracking, Mech. Syst. Signal Process. 21 (2007) 2957-2969.

[27] K.S. Wang, P.S. Heyns, An empirical re-sampling method on intrinsic mode function to deal with speed variation in machine fault diagnostics, Appl. Soft Comput. 11 (2011) 5015-5027.

[28] K.S. Wang, P.S. Heyns, The combined use of order tracking techniques for enhanced Fourier analysis of order components, Mech. Syst. Signal Process. 25 (2011) 803-811.

[29] K.S. Wang, P.S. Heyns, Application of computed order tracking, Vold-Kalman filtering and EMD in rotating machine vibration, Mech. Syst. Signal Process. 25 (2011) 416-430.

[30] M.-C. Pan, C.-X. Wu, Extended angular-velocity Vold-Kalman order tracking, J. Dyn. Sys Meas. Control 132 (3) (2010).

[31] T.W. Parks, C.S. Burrus, Digital Filter Design, John Wiley \& Sons, New York, 1987, Chapter 7.

[32] R.B. R., Cepstrum Analysis, in: Encyclopedia of Vibration, Elsevier, Oxford216-227.

[33] F. La Scala, R. Bitmead, R. James, Conditions for stability of the Extended Kalman Filter and their application to the frequency tracking problem, Math. Control Signals Syst. 8 (1995) 1-26.

[34] F. La Scala, R. Bitmead, Design of an Extended Kalman Filter frequency tracker, IEEE Trans. Signal Process. 44 (3) (1996) $739-742$.

[35] F. La Scala, R. Bitmead, An extended Kalman filter frequency tracker for high-noise environments, IEEE Trans. on Signal Process. 44 (2) (1996) $431-434$.

[36] S. Bittanti, M SavaresiS., On the parameterization and design of an Extended Kalman Filter frequency tracker, IEEE Trans. Autom. Control. 45 (9) (2000) $1718-1724$

[37] H. Hajimolahoseini, M. Reza Taban, H. Soltanian-Zadeh, Extended Kalman Filter frequency tracker for nonstationary harmonic signals, Measurement 45 (2012) 126-132

[38] P. Mohanty, D.J. Rixen, Operational modal analysis in the presence of harmonic excitations, J. Sound Vib. 270 (2004) 93-109. 